\title{
Condiciones de incorporación de las TICs en la Educación Media Técnica en el distrito de Hernandarias
}

\author{
Lucía Esther Villamayor \\ lucia.villamayor@pti.org.py
}

\section{Sady Marinela Prieto González}

sady.prieto@pti.org.py

Fabio López Pires

fabio.lopez@ pti.org.py

PTI-PY-Parque Tecnológico Itaipu Paraguay

\section{RESUMEN}

El presente trabajo de investigación tiene como objetivo determinar cuáles son las condiciones de incorporación de las TICs en la Educación Media Técnica, de gestión pública, del distrito de Hernandarias. Se pretende a través de esta investigación dar respuesta a la siguiente pregunta: ¿Cuáles son las condiciones de incorporación de las TICs en la Educación Media Técnica, de gestión oficial en el distrito de Hernandarias? La metodología implementada fue la investigación evaluativa como método vinculado con el paradigma pragmático o mixto. El diseño de esta investigación es no experimental, y el alcance es descriptivo. La recolección de los datos se llevó a cabo mediante la entrevista y la encuesta. La encuesta estaba conformada por preguntas cerradas y abiertas. La muestra estaba compuesta por 7 (siete) directores de instituciones educativas de gestión oficial, de nivel medio con especialidad en Bachillerato Técnico, en el distrito de Hernandarias, República de Paraguay. Se llegó a la conclusión que las instituciones educativas encuestadas no cuentan con los recursos suficientes para implementar las TIC, la mayoría de los docentes no poseen las competencias necesarias tecnológicas, didácticas y pedagógicas para la incorporación de las TIC y, asimismo, no cuentan, con los recursos tecnológicos para implementarlas.

Palabras clave: Recursos TICs, Docentes, Competencias tecnológicas 


\title{
Conditions of incorporation of ICTs in Technical Secondary Education in the Hernandarias district
}

\begin{abstract}
The present research work aims to determine what are the conditions of incorporation of ICTs in Technical Secondary Education of the Hernandarias district. Through this research, it has been tried to answer the following question: What are the conditions of incorporation of ICTs in Technical Secondary Education public management in the Hernandarias district? The methodology implemented was evaluative research as a method linked to the pragmatic or mixed paradigm. The design of this research is nonexperimental, the scope is descriptive. The data collection was carried out through the interview and the survey. The survey was made up of closed and open questions. The sample consisted of 7 (seven) directors of formally managed educational institutions, of medium level with a specialty in Technical High School, in the district of Hernandarias, Republic of Paraguay. It was concluded that the educational institutions surveyed do not have sufficient resources to implement ICT, most of the teachers do not have the necessary technological, didactic and pedagogical skills for the incorporation of ICT and also do not have the resources technological to implement them.
\end{abstract}

Keywords: ICT resources; Teachers; Technological skills

rtículo recibido: 25 enero 2021 Aceptado para publicación: 28 febrero2021 Correspondencia: lucia.villamayor@pti.org.py Conflictos de Interés: Ninguna que declarar 


\section{INTRODUCCIÓN}

En los últimos años, el sector educativo ha experimentado un proceso de crecimiento en cuanto a incorporación de las TICs en educación. El aumento de infraestructura y conectividad para los centros educativos demuestra el interés del MEC (Ministerio de Educación y Ciencia) en la aplicación de la innovación tecnológica en el proceso de enseñanza aprendizaje. Pero la innovación educativa no es algo que se alcanza inmediatamente, sino que es un proceso, un camino que el docente emprende. En ese proceso se han aplicado algunos programas y/o proyectos como:

El Plan Nacional de Educación 2024 (PNE 2024) que en una de las líneas de acción coloca la Dotación de recursos didácticos y tecnológicos con especial énfasis en las TIC a las instituciones educativas, estudiantes y educadores acordes a los requerimientos de la población/oferta educativa y a estándares de calidad previamente definidos.

Por otro lado, el Proyecto Una Computadora por Niño - One Laptop per Child (OLPC), a través de Paraguay Educa, desde el 2008, implementa TICs como herramientas en el proceso de enseñanza aprendizaje de forma experimental en el Departamento de Cordillera. El plan incluye la dotación de netbooks para alumnos de la Educación Escolar Básica del distrito de Cordillera, asistencia técnica y garantía por dos años, capacitación a los docentes de sus 35 escuelas y un acompañamiento en las áreas de tecnología, desarrollo comunitario y educación.

En el Plan Nacional de Desarrollo 2030 - PND (2014), en el punto que habla sobre Niñez y Adolescencia, plantea Incorporar recursos que permitan acceso a tecnologías en el sistema educativo para reducir la brecha digital y democratización del acceso a la información (STP, 2014, pág. 42). En el mismo documento, en el apartado que se refiere a Investigación, desarrollo tecnológico, innovación y educación superior, propone:

- Fuerte inversión en el despliegue de Tecnologías de la Información y Comunicación, a través de programas nacionales de inclusión digital con las siguientes estrategias: Promoción de iniciativas y aplicación de las tecnologías en la comunidad educativa del país, Programa inclusivo para reducir la brecha digital, Participación de la ciudadanía en la sociedad del conocimiento y Creación de espacios para desarrollar tecnología e innovación” (STP, 2014, pág. 64).

También se tiene el Programa de Mejoramiento de las condiciones de aprendizaje mediante la incorporación de TIC en establecimientos educativos y unidades de gestión 
educativa, en Paraguay, entre los años 2015 y 2018. Este programa forma parte de una iniciativa basada en el Plan Nacional de Educación 2024 y en la Agenda Educativa 2013 - 2018 (MEC, 2013).

Pese a todas estas acciones y avances alcanzados, aún falta mucho para llegar al nivel de inclusión de las TICs como herramientas del proceso de enseñanza aprendizaje. Aparte de eso, la incorporación de las TICs tampoco es garantía del mejoramiento de la educación. Como señala Buckingham (2008), no habrá transformación de la cultura escolar, si la incorporación de las TICs no va acompañada de una mirada pedagógica e innovadora, de las instancias administrativas de la educación.

El problema de estudio de esta investigación está vinculado a las condiciones prácticas de incorporación de las TICs en los procesos de enseñanza aprendizaje en las instituciones educativas de gestión oficial de nivel medio con especialidad en Bachillerato Técnico en el distrito de Hernandarias, República del Paraguay. La investigación busca responder la pregunta: ¿Cuáles son las condiciones de incorporación de las TICs en la Educación Media Técnica, de gestión oficial, del distrito de Hernandarias?; de esta pregunta principal se desprenden las siguientes preguntas específicas: ¿Cuál es la situación de la infraestructura de la institución educativa para la implementación de las TICs?; ¿Cuenta la institución con contenidos educativos desarrollados acorde al programa de estudio del nivel medio técnico?; ¿Tienen los docentes las competencias y capacidades pedagógicas que permitan mejorar la calidad del proceso de enseñanza aprendizaje de los estudiantes, en el marco de la implementación de las TICs?.

Plan Nacional de Educación 2024 -PNE 2014, señala que los docentes constituyen uno de los principales agentes para el mejoramiento de la calidad de la educación (MEC, 2014). La mejora de la calidad de la educación sigue siendo uno de los grandes desafíos de la educación paraguaya.

La investigación plantea como objetivo principal determinar cuáles son las condiciones de incorporación de las TICs en la Educación media Técnica del distrito de Hernandarias. Partiendo de dicho propósito se traza el primer objetivo específico, analizar la situación de la infraestructura de la institución educativa para la implementación de las TICs. El segundo objetivo específico pretende verificar si la institución cuenta con contenidos educativos desarrollados acorde al programa de estudio del nivel medio técnico. Y el tercer objetivo específico busca determinar si los docentes poseen las competencias y 
capacidades pedagógicas que permitan mejorar la calidad del proceso de enseñanza aprendizaje de los estudiantes en el marco de la implementación de las TICs.

El PNE 2024 plantea que la incorporación de las nuevas tecnologías al sistema educativo paraguayo "Aporte al mejoramiento de los procesos educativos a través del uso de las TIC y al desarrollo en todos los estudiantes de las competencias digitales necesarias para participar y contribuir activamente en la sociedad" (MEC, 2014, p. 17). Así también, el PNE 2024 busca generar las condiciones propicias para el desarrollo y sostenibilidad de la implementación de las TICs en la educación, pretende la instalación de las TICs en forma gradual y equitativa, siendo el docente el principal actor de este proceso, priorizando su capacitación en la utilización pedagógica de las TICs en el aula a través de estrategias que avalen la apropiación de las tecnologías por parte de los educadores (MEC, 2014).

Es indiscutible que los cambios científicos y tecnológicos que trae consigo las TICs aparte de los nuevos contextos culturales, influyen en los educadores a formarse en competencias y saberes que anteriormente no precisaban, pues el educador hoy en día cumple funciones mucho más complejas. De ahí la importancia de que los órganos competentes en la formación del profesorado definan nuevas políticas y prácticas en la formación profesional y en la actualización permanente de los docentes.

\section{ESTRATEGIAS METODOLÓGICAS O MATERIALES Y MÉTODOS}

La recolección de la información se llevó a cabo mediante la aplicación de dos instrumentos de recolección de datos; la entrevista y la encuesta. La encuesta estaba basada en la propuesta presentada por el BID, denominada Notas Técnicas ${ }^{1}$, y fue adaptada al contexto de esta investigación estando conformada por preguntas cerradas y abiertas, e incluyó aspectos cuantitativos y cualitativos. Para la validación del instrumento se recurrió al juicio de expertos. Esta forma de validación consiste en la "opinión informada de personas con trayectoria en el tema, que son reconocidas por otros como expertos cualificados en éste, y que pueden dar información, evidencia, juicios y valoraciones" (Escobar-Pérez y Cuervo-Martínez, 2008, p. 29). Por tanto, el instrumento

\footnotetext{
${ }^{1}$ Severin, E. (2010). Tecnologías de la Información y la Comunicación (TICs) en Educación: Marco conceptual e indicadores. Notas Técnicas, Banco Interamericano de Desarrollo, División de Educación (SCL/EDU). Recuperado el 12 de 11 de 2019, de repositorio.minedu.gob.pe/handle/123456789/3394
} 
de recolección de datos se sometió al juicio de expertos en el tema, resultando de ella unas modificaciones en cuanto a la redacción de algunos indicadores, que luego se integró al instrumento final aplicado.

El instrumento se compone de tres variables principales: Infraestructura, Contenidos TICs y Recursos Humanos. Para la aplicación del instrumento fue necesario contar con la autorización escrita de la Dirección Departamental de Educación de Alto Paraná y de las Supervisiones de Apoyo Pedagógico, Región 7, Región 8 y Educación Indígena Zona 2. Para la aplicación de la entrevista a los directores, se elaboró previamente un cuestionario. Al término de cada entrevista se empleó la técnica de la verificación con el participante para asegurar la validez de la información (Valenzuela \& Flores, 2012).

La metodología implementada fue la investigación evaluativa como método vinculado con el paradigma pragmático o mixto, que recoge tanto técnicas cuantitativas como cualitativas (Campoy Aranda, 2016). Según Campoy el paradigma pragmático y el método mixto es un tipo de investigación en el que el investigador o investigadores combinan elementos de abordaje de investigaciones cualitativas y cuantitativas con el objetivo de ampliar y profundizar el conocimiento y su corroboración (p. 123).

Conforme Tamayo (2004) la investigación evaluativa basicamente es un modelo de aplicación de los métodos de investigación para evaluar la eficiencia de los programas de acción en las ciencias sociales. El objetivo de este tipo de investigación es medir los resultados de un programa teniendo en cuenta los objetivos propuestos, con el fin de tomar medidas sobre su proyección y programación para el futuro (p 55). En tanto que Escudero (2011) sostiene que en la investigación evaluativa se debe tener en cuenta el entorno sociopolítico puesto que el campo de intervención se relaciona con las decisiones políticas.

El diseño de esta investigación es no experimental, ya que no manipula variables deliberadamente (Hernández Sampieri, 2010). Es trasversal porque se realizó la recogida de datos en un tiempo determinado y no a lo largo de un continuo. La investigación se encuadra dentro del diseño etnográfico porque pretende describir prácticas de grupos y subsistemas (Hernández Sampieri, 2010, pp. 697).

El alcance es descriptivo, conforme Hernández Sampieri (2010) son aquellos que buscan especificar propiedades, características y perfiles importantes del fenómeno que se somete al análisis, es decir; miden evalúan o recolectan datos sobre diversos aspectos, 
dimensiones o componentes del fenómeno que se investiga. Glass y Hopkins citado por Campoy (2016) menciona que la investigación descriptiva consiste en la recopilación de datos que describen los acontecimientos y luego los organiza, tabula, representa y describe.

La muestra está conformada por 7 (siete) directores de instituciones educativas de gestión oficial, de nivel medio con especialidad en Bachillerato Técnico, en el distrito de Hernandarias, República de Paraguay.

\section{RESULTADOS Y DISCUSIÓN}

Se presenta un análisis de los resultados de la investigación con los datos más destacados teniendo en cuenta la pregunta de investigación ¿Cuáles son las condiciones de incorporación de las TICs en la Educación de gestión oficial, de Nivel Medio, modalidad, Bachillerato Técnico del distrito de Hernandarias? Se presenta en forma ordenada los resultados de las variables Infraestructura, las herramientas tecnológicas y los recursos humanos. En este trabajo de investigación se designa un código para cada institución educativa para salvaguardar la confidencialidad. Quedando denominadas de la siguiente manera: Colegio Técnico 1 - C1, Colegio Técnico 2 - C2, Colegio Técnico 3 - C3, Colegio Técnico 4 - C4, Colegio Técnico 5 - C5, Colegio Técnico 6 - C6, Colegio Técnico 7 - C7.

\section{Infraestructura}

Referente a la variable infraestructura, este trabajo pretende analizar la situación de la infraestructura de la institución educativa para la implementación de las TICs. Para Bates (2001) la infraestructura abarca no sólo la infraestructura física, sino también el apoyo humano, la financiación, la evaluación, la relación entre la infraestructura tecnológica y la planificación académica, el acceso de los estudiantes y del profesorado a la tecnología y el papel de los gobiernos en la ampliación del acceso (Bates, 2001). Esta variable comprende la infraestructura física, los componentes TICs, y la conectividad.

Las principales limitaciones asociadas a la incorporación de TICs están relacionadas a la variable Infraestructura, como la adecuación eléctrica de las instituciones educativas. Esta adecuación de la instalación eléctrica resulta imprescindible para que sea posible la utilización de al menos una sala de informática. Se constató que, de las 07 instituciones educativas, $3(42,86 \%)$ cuentan con medidor de $\mathrm{ANDE}^{2}$ y tomada a tierra. En tanto 4 57:

\footnotetext{
${ }^{2}$ Administración Nacional de Electricidad
} 
14\%) de ellas, tienen conexión directa y no cuentan con medidor de energía, por tanto, son consideradas conexiones irregulares que acarrea consigo un alto grado de peligrosidad para los usuarios por la irregularidad en que encuentra el sistema eléctrico de distribución.

También se verificó la necesidad de redes de comunicación, salas, bibliotecas y muebles necesarios para montar al menos una sala de informática. La investigación realizada permitió detectar que, de 7 instituciones educativas, apenas $3(42,86 \%)$ disponen de conexión de internet. De esta forma los estudiantes no pueden acceder al portal más grande de informaciones que se tiene. El acceso a internet sigue siendo muy limitado para los niños y jóvenes en las instituciones educativas, más aún de zonas rurales.

\section{Gráfico 1: Relación de instituciones educativas con conexión a internet}

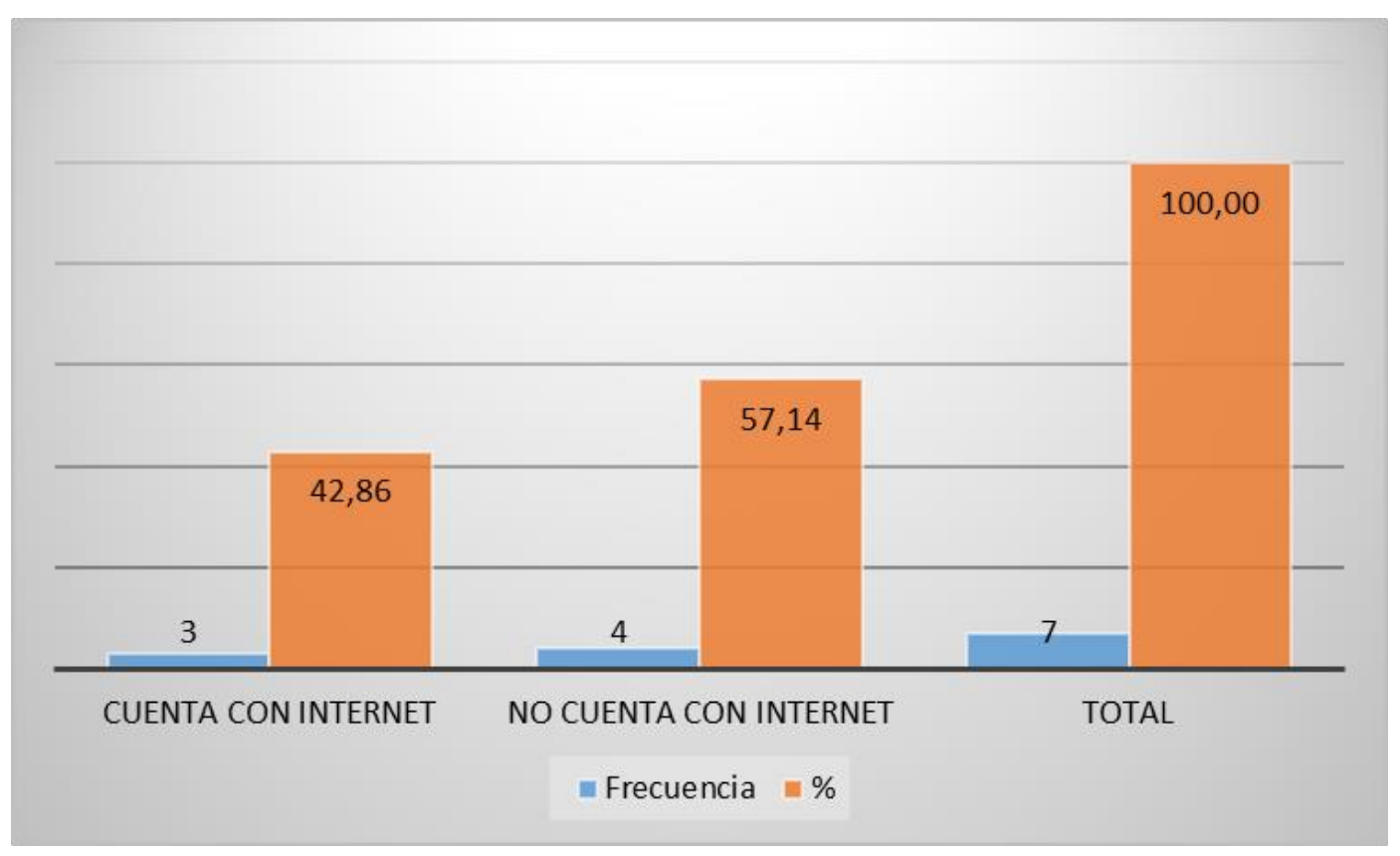

Referente a la cantidad de estudiantes por computadora, esto difiere de una institución a otra conforme la cantidad de estudiantes matriculados. Si se tiene en cuenta el total de estudiantes matriculados (2.518), 173 en zona rural y 2.345 en zona urbana, del total de computadoras en buen estado (32), 31 corresponde a zona urbana y 1 sola computadora para la zona rural. Por tanto, en zona urbana se tienen 75 estudiantes por computadora y en zona rural 173 estudiantes por computadora. 
Gráfico 2: Cantidad de estudiantes por institución educativa en relación a cantidad de computadoras

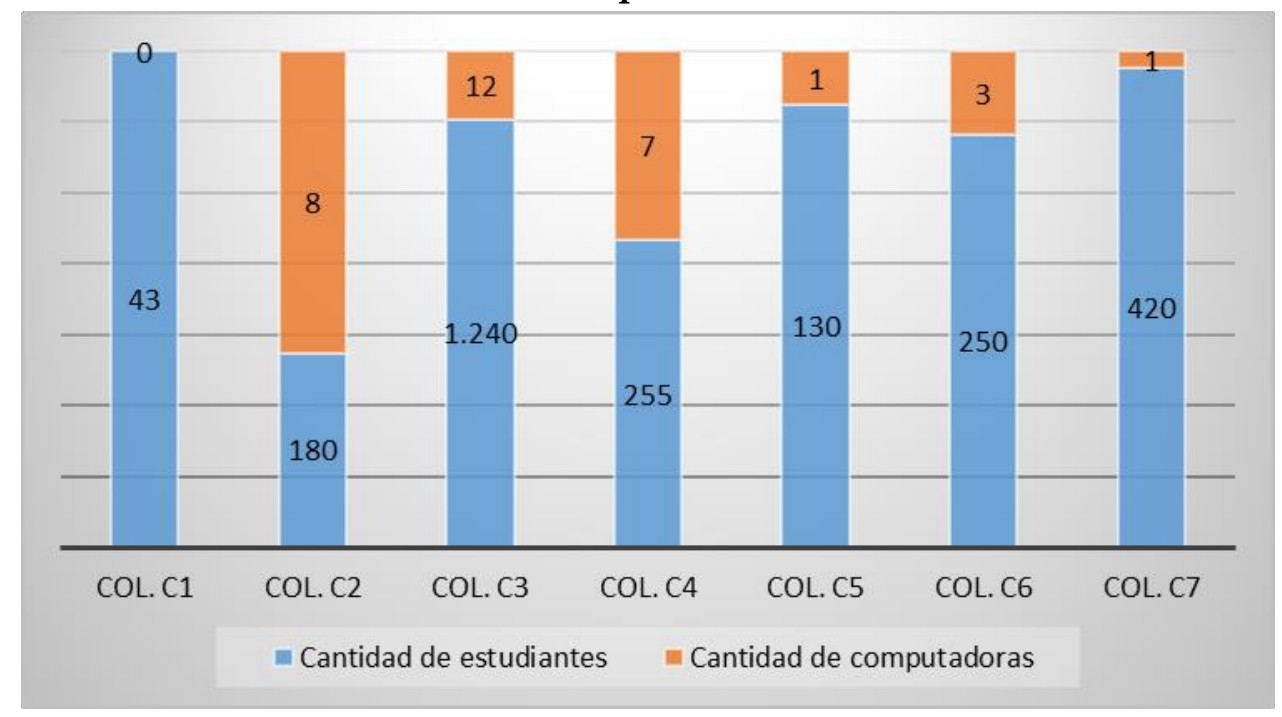

En relación al nivel competitivo de los docentes en las instituciones educativas en el área de informática se verifica que el $18 \%$ tienen conocimiento básico de informática y que implementan las TICs como recurso de aprendizaje.

Referente a los equipamientos audiovisuales - EA con que cuenta la institución educativa para la implementación de las TICs, se tiene que $5(71,43)$ instituciones educativas de las siete encuestadas cuentan con proyector digital, $4(57,14)$ poseen aparato de TV, $3(42,86)$ tienen escáner, 1 (14,29) institución cuenta con cámara fotográfica y $1(14,29)$ con grabadora de video. Ninguna institución cuenta con grabadora de audio, en tanto que una institución educativa indígena reportó que carece de cualquier tipo de equipamientos audiovisuales.

Tabla 1: Equipamientos audiovisuales por Institución Educativa

\begin{tabular}{|l|c|c|c|c|c|c|c|}
\hline $\begin{array}{c}\text { Instituciones } \\
\text { Educativas }\end{array}$ & TV & $\begin{array}{c}\text { Proyector } \\
\text { digital }\end{array}$ & $\begin{array}{c}\text { Reprod. } \\
\text { de } \\
\text { video }\end{array}$ & $\begin{array}{c}\text { Reprod. } \\
\text { de } \\
\text { audio }\end{array}$ & $\begin{array}{c}\text { Cámaras } \\
\text { fotográficas }\end{array}$ & Escáner & Total \\
\hline Col. C1 & 0 & 0 & 0 & 0 & 0 & 0 & 0 \\
\hline Col. C2 & 1 & 2 & 3 & 0 & 0 & 1 & 7 \\
\hline Col. C3 & 1 & 6 & 0 & 0 & 0 & 4 & 11 \\
\hline Col. C4 & 0 & 0 & 0 & 0 & 0 & 0 & 0 \\
\hline Col. C5 & 0 & 1 & 0 & 0 & 0 & 0 & 1 \\
\hline Col. C6 & 1 & 2 & 0 & 0 & 1 & 1 & 5 \\
\hline Col. C7 & 1 & 1 & 0 & 0 & 0 & 0 & 2 \\
\hline
\end{tabular}


En cuanto a la cantidad de computadoras por institución educativa, se encuentra en primer lugar el Col. C3 con 20 computadoras, de los cuales 8 están fuera de servicio por falta de mantenimiento, 14 computadoras $(70 \%)$ son de uso pedagógico y $6(30 \%)$ de uso administrativo. Cabe resaltar que, en este colegio, el Parque Tecnológico de Itaipu Paraguay - PTI-PY montó un laboratorio de informática y se destaca de entre los otros por la cantidad de computadoras que tiene. La relación computadora alumno es de 3 alumnos por cada computadora.

\section{Gráfico 3: Relación de computadoras por institución educativa}

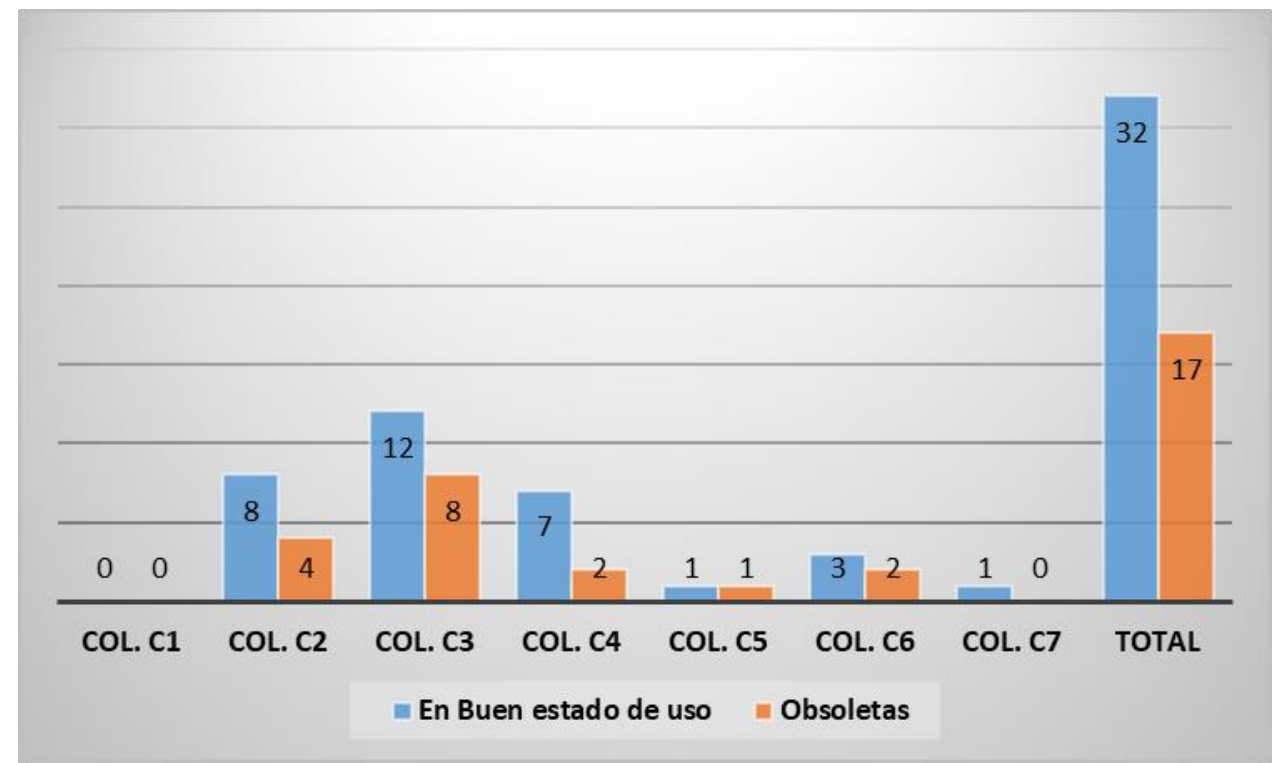

En tanto el Colegio C2 cuenta con 12 computadoras, de los cuales $10(83,33 \%)$ son de uso pedagógico y 2 (16.67\%) de uso administrativo. Cuatro de las computadoras para uso pedagógico están obsoletas. El Colegio C4 posee 9 computadoras, destina 8 (88.89\%) computadoras para uso pedagógico y $1(11.11 \%)$ para el sector administrativo. Dos de las computadoras están fuera de servicio por falta de mantenimiento. En tanto el Colegio C6 tiene 5 computadoras, destina 3 (60\%) computadoras para uso pedagógico y 2 (40\%) para el área administrativa. Como no cuenta con docentes capacitados para el área de informática las computadoras no se usan, y dos computadoras están obsoletas.

El Colegio C7, con especialidad en contabilidad posee una sola computadora y lo usan para el área administrativa. Inclusive la directora usa su propia Notebook para realizar las tareas propias de la institución. La malla curricular del Bachillerato en Contabilidad tiene la materia informática dentro del Plan Específico en el $1^{\circ}, 2^{\circ}$ y $3^{\circ}$ año, pero como la institución carece de computadoras se administra solo en su contenido teórico. El Colegio 
C5 tiene 2 computadoras que son destinadas exclusivamente para uso administrativo. Una de las computadoras está con muchos problemas por falta de mantenimiento. Una sola computadora es la que está en óptimas condiciones de uso. En tanto el Colegio C1 no posee ninguna computadora.

Del total de computadoras registradas (49), el 65,31\% están en buen estado de uso, y el $34,69 \%$ son reportadas como obsoletas. (Ver gráfico 4).

\section{Gráfico 4: Relación de computadoras en funcionamiento y obsoletas}

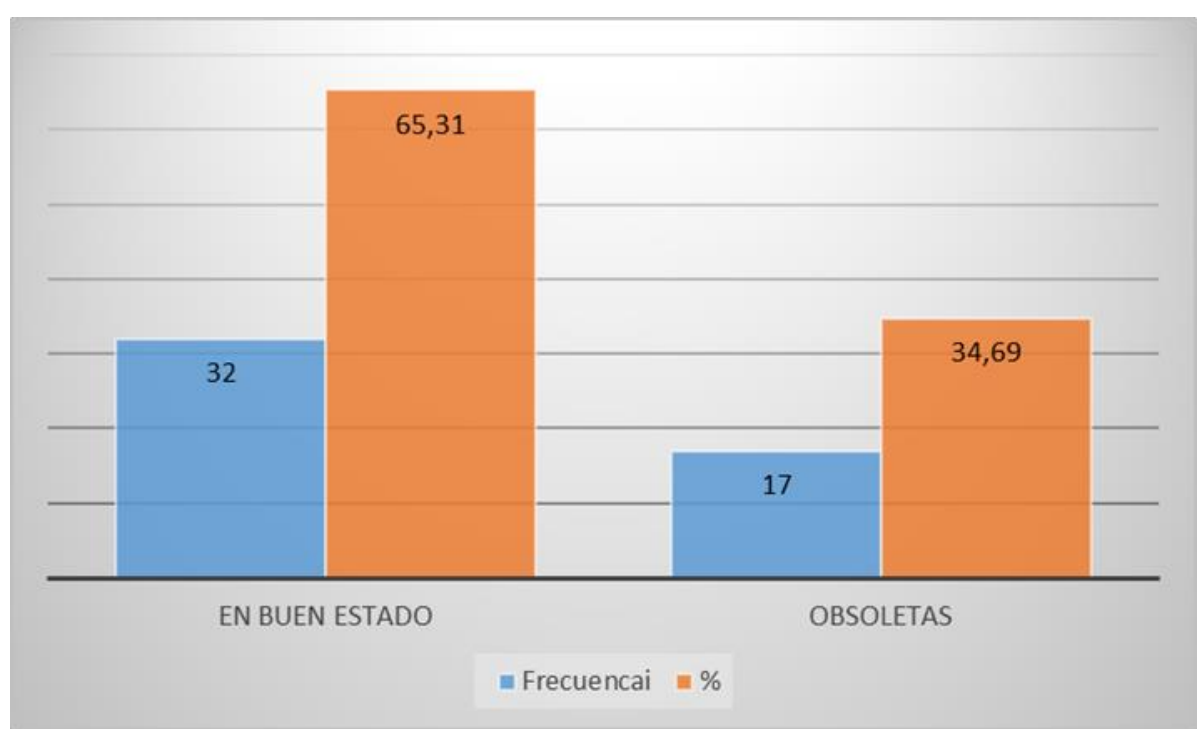

Ninguna de las instituciones encuestadas tiene personal técnico de planta para el atendimiento técnico de los equipos informáticos. El Col.C3 y el Col. C6 reportaron que puntualmente contratan soporte técnico externo a través de las Asociaciones de Cooperación Escolar (ACEs).

Los problemas de los equipos informáticos reportados se reducen a la falta de mantenimiento y falta de técnicos en informática para dar mejor uso a los equipos. La mayoría de las computadoras existentes en las instituciones tienen entre 5 y 8 años de uso, muchas de ellas han caído en desuso por falta de mantenimiento.

A través de esta investigación se observó que la mayoría de las instituciones educativas encuestadas no tienen la cantidad necesaria de equipos informáticos y espacios físicos adecuados para que los docentes puedan ejecutar las actividades pedagógicas con los estudiantes.

\section{Contenidos}


En relación a la variable contenidos, se busca analizar si la institución cuenta con contenidos educativos desarrollados acorde al programa de estudio del nivel medio técnico.

Los cursos del Nivel Medio Técnico que reciben cursos de conocimientos básicos de informática según se prescriben en el currículo en los tres años de la Educación Media son los estudiantes del curso Técnico en Contabilidad, Técnico en Hotelería y Turismo, Técnico en Electricidad, Técnico en Electrónica y Técnico en Química Industrial.

Se constató que apenas 2 (29\%) de los colegios encuestados implementan ejercicios por computadora y utilizan software educativo e internet, mientras que 5 (71\%) no implementan ejercicios por computadora. El Col. C3 es la única institución educativa que utiliza Internet para hacer investigaciones y ejercicios en computadora. El currículo tiene un Plan Optativo de informática que la institución utiliza para incorporar las TICs como instrumento de experimentación y construcción de contenidos.

Del total de siete instituciones educativas, solo 3(42,86\%) implementan las TICs en el proceso de enseñanza aprendizaje.

\section{Gráfico 5: Instituciones Educativas que implementa las TICs en el proceso de enseñanza aprendizaje}

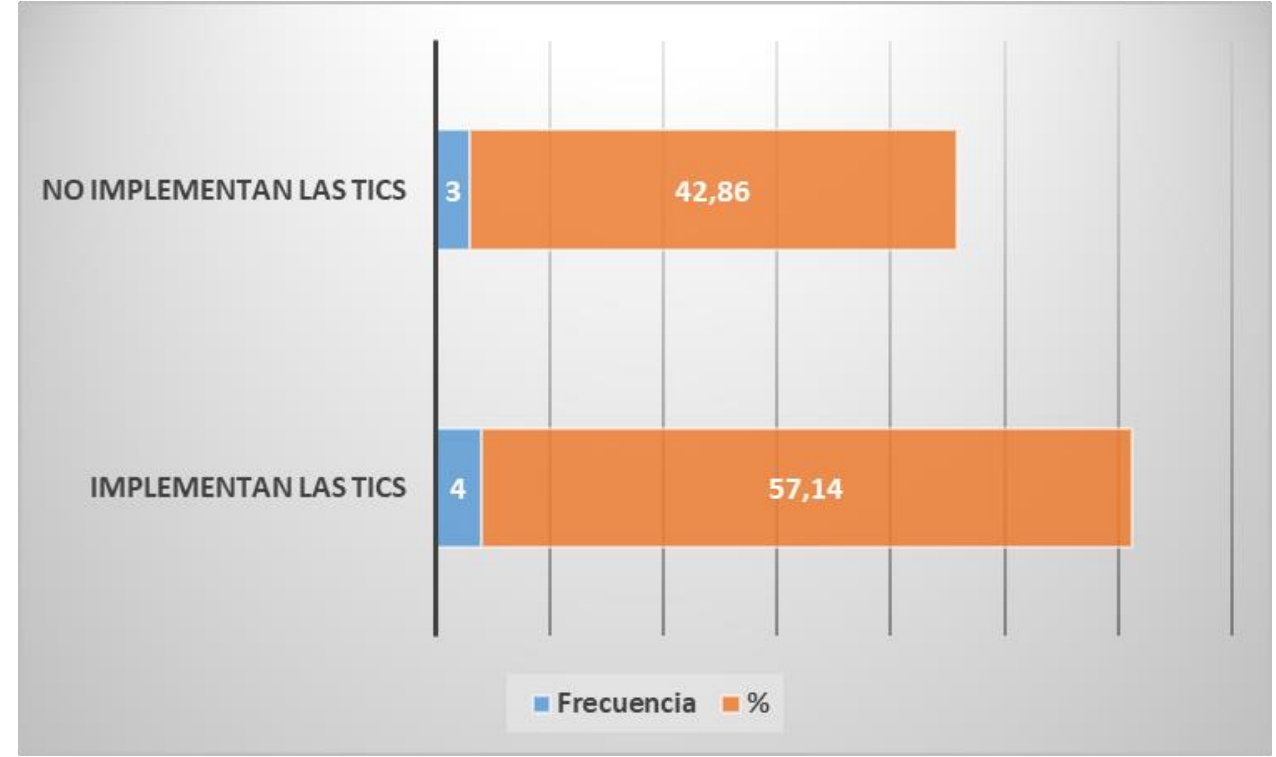

En cuanto a la pregunta sobre innovación curricular para el uso de las TICs con fines educativos el director del Col. C2 indicó que el currículo presentado por el $\mathrm{MEC}^{3}$ es innovador, pero no se tiene el apoyo necesario para llevar a la práctica, ya que no se

${ }^{3}$ Ministerio de Educación y Ciencia 
cuenta con internet para utilizar las diferentes plataformas educativas, ni siquiera se cuenta con medidor de ANDE. No se puede hacer ningún tipo de trabajo de investigación usando internet. Algunas instituciones educativas no cuentan con infraestructura necesaria para implementar las innovaciones. Cabe recordar que varias de las instituciones no cuentan con computadoras ni Internet.

Referente al número promedio de horas reloj semanales destinadas al uso de las TICs en el aula según se prescribe en el currículo del Nivel Medio Técnico se tiene una media de 2 horas semanales. En una de los colegios las clases son meramente teóricas porque carecen de equipos informáticos.

\section{Recursos Humanos}

En cuanto a Recursos Humanos, con preparación suficiente para la implementación de las TICs, se percibe que pocos docentes tienen la preparación adecuada para implementar las TICs. Apenas el 18\% de los docentes de las instituciones educativas encuestadas están capacitados para enseñar utilizando las TICs.

Si cruzamos los datos con la cantidad de docentes por institución y los que están certificados para enseñar conocimientos básicos de informática en secundaria se nota una diferencia bastante llamativa que puede apreciarse en el siguiente gráfico.

Gráfico 6: Estudiantes y docentes con conocimiento de informática

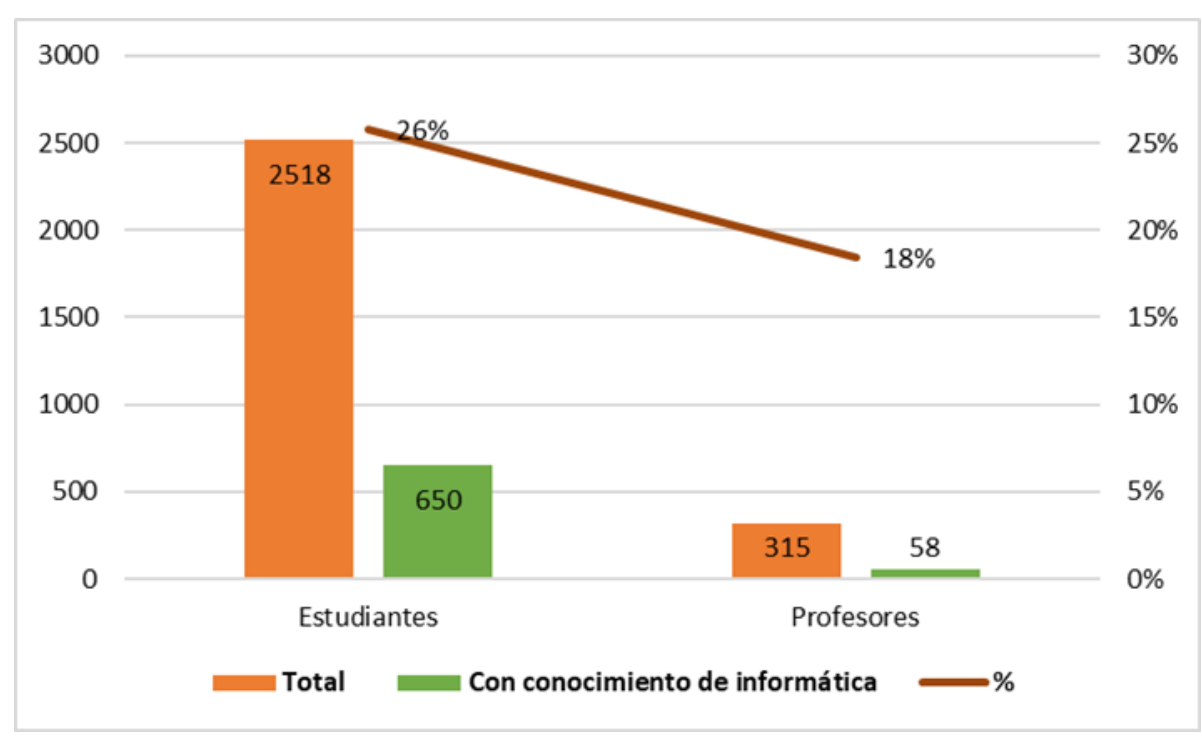

En cuanto al apoyo pedagógico profesional explícito a los docentes para la incorporación de TICs al currículum, el 100\% de las instituciones encuestadas respondieron que carecen de cualquier tipo de apoyo pedagógico para la incorporación de las TICs al currículo. Cada docente procura bajar a su realidad conforme pueda. 
Además de la falta de equipamientos y soporte técnico los directores también manifestaron la falta de los conceptos pedagógicos claros para utilizar efectivamente las TICs de manera a que favorezca el mejoramiento de los aprendizajes de los estudiantes

\section{CONCLUSIÓN O CONSIDERACIONES FINALES}

Esta investigación estuvo enfocada exclusivamente en directores de Instituciones Educativas de Gestión Oficial de Nivel Medio con Modalidad Bachillerato Técnico del distrito de Hernandarias. Las conclusiones alcanzadas tienen un alcance limitado, sin embargo, muchas instituciones educativas de Paraguay presentan características semejantes a las encontradas en este trabajo, por lo tanto, podría ser una contribución en el avance del uso de las TICs en el proceso de enseñanza aprendizaje.

Al término de investigación, se percibe que buena parte del cuerpo docente de las instituciones educativas encuestadas, presentan limitaciones en cuanto a formación pedagógica en las áreas de las TICs. Esto constituye un importante factor que restringe la introducción de innovaciones didácticas tecnológicas.

Además, se constató la falta de infraestructura para la implantación de las TICs. Falta de equipos informáticos y mobiliarios. Por lo demás, no basta con tener computadoras, TV, reproductor de audio y video, si no se cuenta con una red de internet.

La falta de capacitación de los docentes en los diversos aspectos de TICs es uno de los mayores problemas encontrados. Los docentes mantienen una práctica pedagógica tradicional, donde el conocimiento es visto como la información que el docente debe trasmitir y el alumno debe asimilar pasivamente sin pasar por el proceso de construcción colectiva del conocimiento.

Es importante que los docentes logren niveles óptimos de interacción curricular utilizando los softwares educativos, para ello se necesitará proporcionar formación en el uso de las TICs a los docentes. Solo así podrán dar un uso educativo a las tecnologías de la información y podrán utilizarlo en los procesos de enseñanza-aprendizaje.

Conforme la directora del colegio C5, el docente muchas veces no posee ni los conocimientos básicos, y, menos aún, las competencias básicas en cuanto a informática. La directora del $\mathrm{C} 1$ expresó que no todos los docentes pueden cambiar sus prácticas educativas, por más que quisieran hacerlo, por causa del desconocimiento que muchos docentes tienen respecto a las TICs, especialmente, los que están en zonas rurales. 
Se puede decir que las actitudes de los profesores determinan en cierta forma el grado de implementación de las TICs, ya que la modificación de la práctica tradicional de enseñanza aprendizaje genera cierto tipo de desconfianza y rechazo por el desconocimiento y, en ciertos casos también, vergüenza por esa inexperiencia.

Cabe resaltar también, que las situaciones didácticas de la implementación de las TICs en las instituciones educativas objeto de análisis en este trabajo, no aparecen debidamente estructuradas, pues todos los directores respondieron que carecen de coordinador de TICs, y de orientación pedagógico didáctica en el uso de las TICs.

En tanto la directora del Col. C6. manifestó, además de que el currículo del Bachillerato Técnico Agropecuario no contempla las clases de informática, no cuentan con profesores de informática, y tampoco los docentes tienen interés en utilizar las computadoras, porque para eso deben instalar data show y trasladar a otra sala a los estudiantes, y los profesores catedráticos lo consideran una pérdida de tiempo, pues tienen el tiempo contado para llegar a otra institución. Decía la directora que esos docentes "son los llamados profesores taxi” porque son catedráticos, y tienen clases en diferentes instituciones educativas. El Col. C6 cuenta con infraestructura y equipos, sin embargo, no tiene los recursos humanos capacitados para poder implementar el uso de las TICs, ni tan siquiera para pasar un PPS. Pese a existir políticas claramente definidas sobre la incorporación de las TICs en la educación, aún no se implementan de forma eficiente desde la gestión propia del programa. Se percibe una reducida incorporación a lo que cada docente pueda llegar a desarrollar según su iniciativa. Por ende, el uso e implementación de las TICs, en el proceso de enseñanza aprendizaje, se presenta en una dimensión muy básica frente al gran potencial que tienen estas herramientas en el ambiente educativo.

Los números son bastante ilustrativos de la situación, pues solo el 42,86\% de instituciones educativas encuestadas tienen Internet. El 100\% de las instituciones educativas tienen acceso a energía eléctrica, pero solo el $42,86 \%$ cuentan con medidor de energía, por lo tanto, el $57,14 \%$ tiene conexión directa sin medidor, serían conexiones irregulares que acarrean consigo un alto nivel de peligrosidad teniendo en cuenta la posible ocurrencia de accidentes por electrocución o incendios por el estado en el que se encuentra el sistema eléctrico de distribución.

El $42,86 \%$ de las instituciones encuestadas no tiene clases de informática, en tanto el 14,29\% tiene clase de informática teórico práctico y el 42,86\% solo tiene clases teóricas 
de informática por falta de computadoras. En cuanto a cantidad de computadoras por estudiante, la relación es de 70 estudiantes por computadora. El 57,14\% de los colegios no tienen soporte técnico, en tanto, $42,86 \%$ tienen soporte técnico particular en forma eventual. El 71,43\% de las computadoras, son de uso pedagógico y el 28,57\% de uso administrativo. Del total de computadoras existentes, 34,69\% son obsoletas.

Las principales dificultades encontradas para la incorporación de las TICs en las instituciones educativas encuestadas se podrían resumir en:

- Falta de infraestructura TICs adecuada en las instituciones educativas de nivel técnico (computadoras, acceso a internet, sistemas de información)

- Escaso o nulo mantenimiento de los equipos existentes (falta soporte técnico, asistencia remota, y estrategias de renovación de los equipos informáticos)

- Bajo nivel de utilización de las TICs por los docentes para uso pedagógico a pesar de que están disponibles.

- Falta de capacitación docente en el uso pedagógico de las TICs.

- Insuficiente acompañamiento pedagógico al docente en la implementación de las TICs

Para concluir se puede decir que el problema no está solo en el uso o no de las TICs en el proceso de enseñanza aprendizaje, sino en el diseño, desarrollo y evaluación del currículo que implementa las TICs y de su uso como medio de aprendizaje y no como fin y en la formación pedagógica, didáctica y técnica del docente en el uso de las TICs.

\section{LISTA DE REFERENCIAS}

Bates, T. (2001). Cómo gestionar el cambio tecnológico, Barcelona, Editorial Gedisa/Ediciones UOC. (E. UOC, Ed.) Barcelona: Editorial Gedisa.

Buckingham, D. (2008). Más allá de la tecnología. Aprendizaje infantil en la era de la cultura digital. Buenos Aires: Manantia.

Campoy Aranda, T. (2016). Metodología de la Investigación Científica: Manual para la elaboración de tesis y trabajos de investigación. Asunción: Marben.

Escobar-Pérez, J., \& Cuervo-Martínez, A. (2008). Validez de contenidos y juicios de expertos: una aproximación a su utilización. Avances en Medición, 6(27-36). $\begin{array}{lllllll}\text { Recuperado } & \text { el } & 10 & \text { de } & 12 & \text { de }\end{array}$ ttp://www.humanas.unal.edu.co/psicometria/files/7113/8574/5708/Articulo3_Jui cio_de_expertos_27-36.pdf 
Escudero, T. (2011). La construcción de la investigación evaluativa. El aporte desde la educación. Zaragoza: Prensas Universitarias de Zaragoza.

Hernández Sampieri, R. F. (2010). Metodología de la investigación. México: McGrawHill.

Hung, E. S. (2015). Hacia el fomento de las TIC en el sector educativo en Colombia. Colombia: Universidad del Norte.

Johnson, R. B. (2007). Toward a definition of mixed methods research. Journal of Mixed Methods Research, 1(2), 112-133.

MEC. (2013). Incorporación de TIC en el Sistema Educativo Nacional. Asunción.

MEC. (2014). Plan Nacional de Educación 2024. Hacia el centenario de la Escuela Nueva de Ramón Indalecio Cardozo. Asunción.

Sarmiento, S. R. (2015). Factores que inciden en la implementación de las TIC en los procesos de enseñanza-aprendizaje en $5^{\circ}$ de Primaria en Colombia. Revista Complutense de Educación, 26(Especial), 197-213.

Severin, E. (2010). Tecnologías de la Información y la Comunicación (TICs) en Educación: Marco conceptual e indicadores. Notas Técnicas, Banco Interamericano de Desarrollo, División de Educación (SCL/EDU). Recuperado el 12 de 11 de 2019, de repositorio.minedu.gob.pe/handle/123456789/3394

STP. (2014). Plan Nacional de Desarrollo Paraguay 2030. Asunción: Secretaría Técnica de Planificación-STP.

Tamayo, M. (2004). El proceso de la investigación científica. Limusa.

Trucano, M. (2005). Knowledge Maps: ICTs in Education. Washington, D.C.: infoDev/The World Bank. Recuperado el 28 de 11 de 2019, de http://www.infodev.org/infodev-files/resource/InfodevDocuments_8.pdf

Valenzuela, J. R., \& Flores, M. (2012). Fundamentos de investigación educativa (Vol. II). Monterrey, Nuevo León, México: Editorial Digital del Tecnológico de Monterrey. 